15

\title{
Пространственное и энергетическое формирование нейтронных пучков с помощью гибких фторполимерных нейтроноводов
}

\author{
(C) С.С. Арзуманов, В.И. Морозов, Ю.Н. Панин, А.Н. Стрепетов \\ Национальный исследовательский центр „Курчатовский институт“, \\ 123182 Москва, Россия \\ e-mail: Arzumanov_SS@nrcki
}

(Поступило в Редакцию 30 августа 2016 г.)

\begin{abstract}
Представлены результаты экспериментов по пространственному отклонению и энергетическому формированию реакторных нейтронных пучков с помощью гибких полихлорвиниловых трубок, внутренняя поверхность которых покрыта тонким слоем жидкого фторполимера.
\end{abstract}

DOI: 10.21883/JTF.2017.03.44260.2023

В работах [1-2] было обнаружено исключительно высокое пропускание ультрахолодных нейтронов (УХН) полихлорвиниловыми трубками промышленного производства. Полихлорвинил (ПВХ) представляет собой высокомолекулярный полимер $\left(\mathrm{CH}_{2}-\mathrm{CHCl}\right)_{n}$ с высоким молекулярным весом молекул в пределах от 30000 до $100000 \mathrm{amu}$. Граничная скорость полихлорвинила $V_{\lim }=2.9 \mathrm{~m} / \mathrm{s}$, поэтому нейтроны, падающие на гладкую поверхность с нормальной компонентой скорости $V_{n}<V_{\text {lim }}$, зеркально отражаются. Величину $V_{\lim }$ можно увеличить, если на поверхность полихлорвинила нанести тонкий $(\sim 10 \mu \mathrm{m})$ слой вещества, обладающего более высокой граничной скоростью, но сохраняющего зеркальность поверхности. В качестве такого покрытия

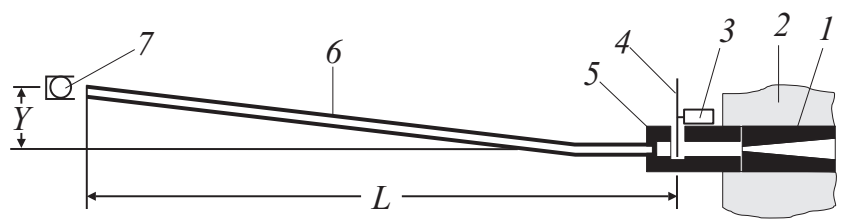

Рис. 1. Схема эксперимента: $1-$ стальной конический коллиматор, 2 - биологическая защита, 3 - двигатель диска прерывателя пучка, 4 - диск-прерыватель пучка, 5 - выходной коллиматор из борированного полиэтилена, $6-$ ПВХ трубка, 7 - счетчик медленных нейтронов „Гелий-18/180-8.0 БЦ“.

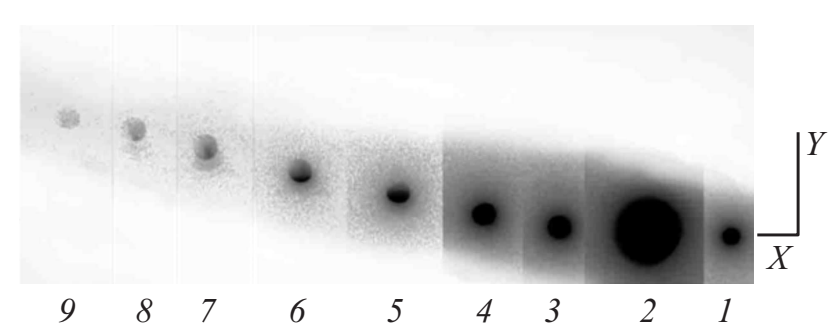

Рис. 2. Изображения нейтронного пучка, полученные с помощью Image Plate детектора: 1 - на выходе коллиматора из борированного полиэтилена, $2-$ на расстоянии $L=118 \mathrm{~cm}$, 3 - на выходе прямой трубки, 4-9 - на выходе изогнутой трубки при $Y=4,8,12,16,20,24 \mathrm{~mm}$ соответственно. может быть использован жидкий фторполимер Fomblin YL VAC 18/8, увеличивающий граничную скорость до $4.56 \mathrm{~m} / \mathrm{s}$. Коэффициент поглощения нейтронов при отражении от слоя не превышает $(2-3) \cdot 10^{-5}$.

ПХВ трубки с жидким фторполимерным покрытием могут быть использованы для пространственного и энергетического формирования реакторных нейтронных пучков. Для тепловых нейтронов со скоростью $2200 \mathrm{~m} / \mathrm{s}$ отражение происходит, когда нейтроны падают на поверхность под углом, меньшим критического: $\theta<0.0021$. При уменьшении скорости нейтронов критический угол отражения возрастает. Нейтроны, проникшие в слой фторполимера, либо рассеиваются, либо захватываются на ядрах. Поэтому по прямой цилиндрической трубке за счет отражений может распространяться поток нейтронов, у которых осевая $V_{z}$ компонента скорости произвольна, а $V_{n}<4.56 \mathrm{~m} / \mathrm{s}$. Изгиб трубки без существенной потери интенсивности прошедшего пучка возможен, пока выполняется условие $(2 d / R)^{0.5}<\theta$, где $R$ - радиус изгиба трубки, $d-$ внутренний диаметр. Таким образом, изогнутая цилиндрическая трубканейтроновод может изменять как направление пространственного распространения пучка тепловых нейтронов, так и его спектральный состав. В настоящей работе описаны возможности отклонения нейтронных пучков и их энергетического формирования с помощью таких трубок.

Схема измерений энергетического распределения нейтронов на выходе трубки методом времени пролета представлена на рис. 1. При исследовании возможности пространственного отклонения нейтронного пучка диск-прерыватель 4 удалялся, а детектором нейтронов 7 служила пластина позиционно-чувствительного Image Plate детектора.

Для измерений использовался касательный горизонтальный канал № 7а реактора ИР-8 НИЦ „Курчатовский институт". В этом канале источником нейтронов является дополнительный бериллиевый рассеиватель нейтронов, установленный вблизи активной зоны реактора на расстоянии $5.4 \mathrm{~m}$ относительно выхода пучка. Нейтроны 


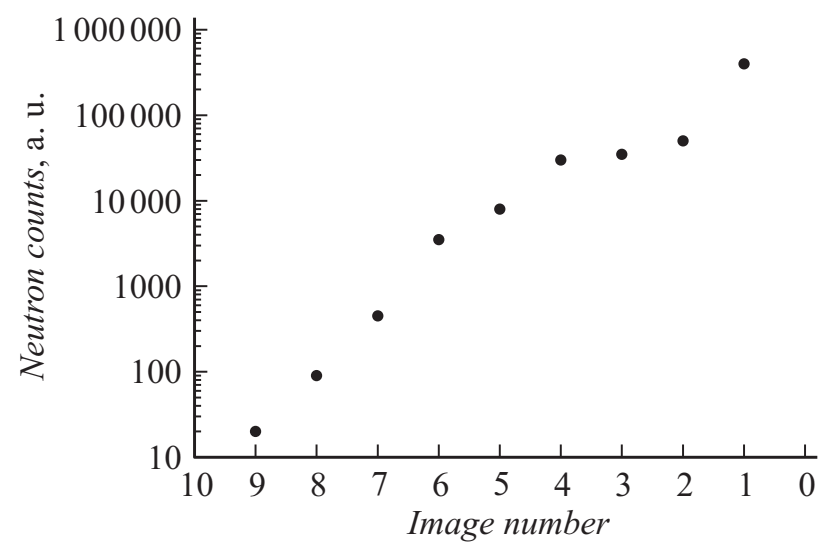

Рис. 3. Значения максимальной плотности потока нейтронов в полученных изображениях. из рассеивателя проходили через первый конический стальной коллиматор с входным диаметром $80 \mathrm{~mm}$, выходным $40 \mathrm{~mm}$ и длиной $2 \mathrm{~m}$ (на рис. 1 не показан). Далее нейтроны поступали во второй выходной конический коллиматор из стали с входным диаметром $8 \mathrm{~mm}$, выходным диаметром $3 \mathrm{~mm}$ и длиной $70 \mathrm{~cm}$. На выходе этого коллиматора формировался пучок нейтронов с угловой расходимостью около $1^{\circ}$. Далее нейтроны поступали во вспомогательный коллиматор из борированного полиэтилена, в котором крепился начальный участок гибкой ПВХ трубки. Трубка имела внутренний диаметр $8 \mathrm{~mm}$, внешний $-14 \mathrm{~mm}$, длину $L=118 \mathrm{~cm}$. Внутренняя поверхность покрыта тонким слоем жидкого фторполимера Fomblin YL VAC 18/8. Трубка устанавливалась горизонтально по оси пучка,
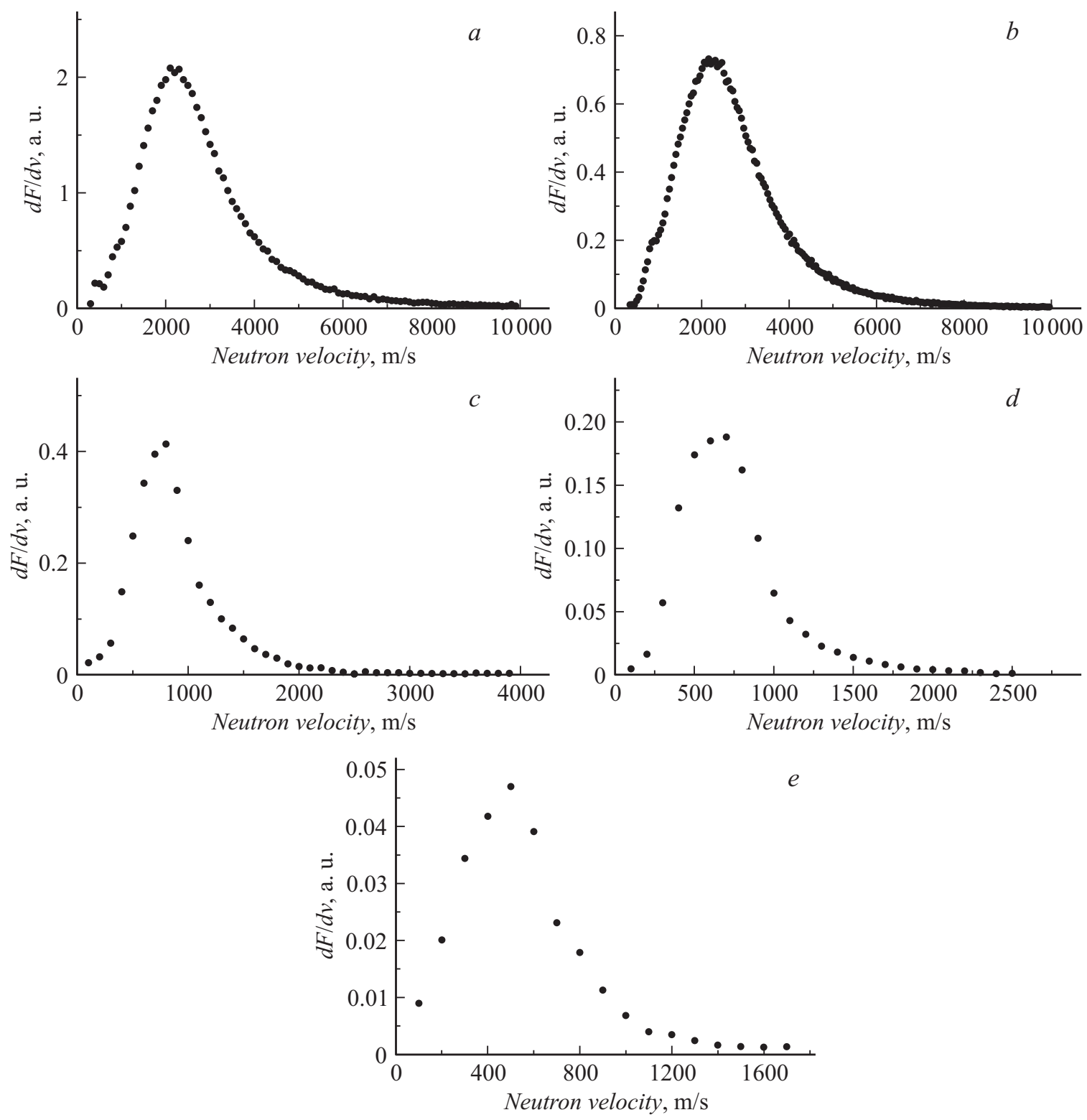

Рис. 4. Спектры потока нейтронов $d F / d v$ в зависимости от скорости нейтронов $v$ при различных высотах $Y$ подъема выходного конца трубки: $a-$ без трубки, $b-e-$ на выходе трубки при $Y=0,6,12,18 \mathrm{~mm}$ соответственно. 
затем плавно изгибалась вверх на начальном участке длиной 50-100 mm. При этом остальная часть трубки оставалась прямой, а выходной ее конец поднимался на высоту $Y$, изменявшуюся от 0 до $40 \mathrm{~mm}$.

На рис. 2 показаны оптические изображения нейтронного пучка, полученные с помощью Image Plate детектора, чувствительная пластина которого располагалась вблизи выходного отверстия трубки. Распространение нейтронов по прямой трубке происходит как за счет прямого пролета, так и за счет упругих отражений от стенок. Для обеспечения одинаковой эффективности регистрации использовалась одна и та же пластина, перемещавшаяся перпендикулярно оси пучка в горизонтальном направлении примерно на $25 \mathrm{~mm}$ при каждой следующей экспозиции.

На выходе коллиматора из борированного полиэтилена нейтронный пучок имеет диаметр около $5 \mathrm{~mm}$ (изображение 1 ), проходя расстояние $L=118 \mathrm{~cm}$, пучок расширяется до диаметра около $21 \mathrm{~mm}$ (изображение 2). При установке на пучок прямой трубки длиной $118 \mathrm{~cm}$ трубка формирует на выходе пучок диаметром $8 \mathrm{~mm}$, равный ее внутреннему диаметру (изображение 3). При отклонении более чем на величину внутреннего диаметра трубки $(Y>8 \mathrm{~mm})$ выходящий поток обусловлен уже только нейтронами, отражающимися от ее внутренней поверхности (изображения 5-9). Это свидетельствует о том, что исследуемые трубки позволяют эффективно отклонять нейтронные пучки с энергией, близкой к тепловой.

О потерях интенсивности отклоняемых пучков можно судить по рис. 3, где представлены значения максимальной плотности потока нейтронов в полученных изображениях. Номер каждого значения соответствует номеру изображения на рис. 2 .

Исследование спектральных изменений нейтронного потока на выходе трубок проводилось время-пролетным методом. Поступающий в трубку поток тепловых нейтронов проходил через щель шириной $1 \mathrm{~mm}$ во вращающемся кадмиевом диске диаметром $200 \mathrm{~mm}$. Частота оборотов составляла $12.5 \mathrm{~Hz}$. Осевая компонента скорости нейтронов (практически совпадающая с полной скоростью) анализировалась по времени их пролета базового расстояния, практически совпадающего с длиной трубки. На рис. 4 представлены измеренные спектры потока нейтронов $d F / d v$. При измерениях с прямым потоком без трубки и с прямой трубкой спектр потока описывется распределением, близким к максвелловскому с наиболее вероятной скоростью $2300 \mathrm{~m} / \mathrm{s}$. При подъеме выходного конца трубки на высоту $Y=6 \mathrm{~mm}$ наиболее вероятная скорость проходящего через трубку потока нейтронов уменьшается до $800 \mathrm{~m} / \mathrm{s}$ и в спектре практически исчезают нейтроны с скоростью более $2200 \mathrm{~m} / \mathrm{s}$. При подъеме трубки на высоту $Y=12 \mathrm{~mm}$ наиболее вероятная скорость проходящего потока уменьшается до $700 \mathrm{~m} / \mathrm{s}$, а при подъеме на высоту $Y=18 \mathrm{~mm}$ наиболее вероятная скорость составляет 500-600 m/s. При этом в спектре проходящего потока практически отсутствуют нейтроны со скоростью более $1600 \mathrm{~m} / \mathrm{s}$.

Таким образом, гибкие ПХВ трубки с жидкостным фторополимерным покрытием позволяют производить пространственное и энергетическое формирование реакторных пучков, что представляет большой интерес для фундаментальных и практических нейтронных исследований. При дальнейшем совершенствовании трубкинейтроноводы могут быть использованы для извлечения нейтронов холодного и ультрахолодного диапазонов из низкотемпературных конверторов. С практической точки зрения возможно применение трубок как нейтронных катетеров для прямой доставки тепловых нейтронов к злокачественным опухолям при лечении методом нейтрон-захватной терапии [3]. При этом применение гибких нейтроноводов позволяет проводить облучение без поражающего здоровые ткани организма воздействия быстрых нейтронов и $\gamma$-излучения ядерного реактора.

\section{Список литературы}

[1] Arzumanov S., Morozov V., Panin Yu. et al. // ISINN-XYIII. Proceedings of the Seminar. Dubna, 2011. P. 17-22.

[2] Arzumanov S.S., Bondarenko L.N., Geltenbort P.W. et al. // Crystallography Rep. 2011. V. 56. N 7. P. 1197-1199.

[3] Арзуманов С.С., Бондаренко Л.Н., Загрядский В.А. и др. // Атомная энергия. 2010. Т. 109. Вып. 1. С. 19-28. 